\title{
THE RECOGNITION OF HERITAGE QUALITIES FROM FEATURE-BASED DIGITAL PROCEDURES IN THE ANALYSIS OF HISTORICAL URBAN CONTEXTS
}

\author{
R. De Marco ${ }^{1}$, A. Pettineo ${ }^{1}$ \\ ${ }^{1}$ DICAr, Dept. of Civil Engineering and Architecture, University of Pavia, Italy \\ raffaella.demarco@unipv.it, alberto.pettineo01@universitadipavia.it
}

\author{
Commission II
}

KEY WORDS: urban historical heritage, multi-source digital survey, urban semantics, feature regions, Upper Kama.

\begin{abstract}
:
The documentation of historical architectural heritage in urban contexts involves the consideration of planning adaptations of settlements and landscape, related to the identification of formal and semantic qualities. In particular, the identification of cultural significance of Heritage building units can find correspondence in geometrical features that are documented within the urban asset. In this way, urban monitoring, in an increasingly automated way, can support the identification and characterization of semantic elements also regarding Heritage objects, observing the invariance and conservation of formal constants in urban dynamic assets. Considering the experimental case study of Solikamsk historical center, belonging to Upper Kama route (Russia), a multiinstrumental strategy of spatial survey is applied, evaluating data coverages and resolutions. This analysis defines a preliminary framework to develop further processes of $3 \mathrm{D}$ triangulation and reality-based meshing. The morpho-metric detail of final models constitutes the basis for the computing test of feature-based procedures, including regions recognition and mesh segmentation, which can be calibrated for shape qualities and scales, reaching a preliminary modeling classification of Heritage and urban building units.
\end{abstract}

\section{INTRODUCTION}

\subsection{Spatial documentation concerning Heritage assets}

The topic of documenting historical Built Heritage involves, in a common percentage of cases, the location of the site in an urban surrounding context, usually shaped as a historic centre or a densely built area. In the case of isolated sites within the settlements, even connected to each other by common characters (e.g., type, function, significance), the location scale can often be wider, till to reach the territorial one.

In an urban/territorial asset, a spatial documentation process considers social-infrastructural characteristics that are inevitably included from the configuration of the settlement structure to the spatial documentation of the building itself. In this way, the architectural documentation cannot be conceived as separated from the physical consistency of the surrounding structures.

The urban spatial context is supposed to include both static elements (e.g. residential buildings, urban design assets, geological and hydrological structures, territorial paths) and dynamic systems (e.g. traffic, public flows, temporary structures, vehicles), Regarding the dynamic ones, they concern services and functional features of the city, and their monitoring is mainly applied through the use of motion sensors, able to reference the spatial data to a "temporal" query for translating the unclassified recordings into significant datasets.

Regarding static features of the urban environment (as long as we can use the term "static" within the continuous planning evolvement of the ecosystem of a city), different methods and instrumental applications of territorial spatial survey can be listed (i.e. remote sensing, topography, satellite positioning systems, terrestrial or aerial photogrammetry, laser scanning systems). Each one inevitably influences the type and tolerance of recognition of the categories of urban elements, including the analysis of geometrical details and building qualities.

Considering the characterization of historical buildings within the urban context, the pre-eminence of Heritage objects is well distinguishable. The historical design of architectural units, joint with the cultural significance of the building itself and of its apparatuses, suggests the evaluation of different tolerances for the interpretation and classification of spatial qualities in 3D survey datasets (i.e., point clouds) between built heritage and urban units. Physical dimensions, geometrical proportions, and shape resolutions can be quantified differently between the Heritage objects and Urban units, thus conceiving a different qualification of territorial mapping results.

In this way, the monitoring of heritage features within the urban ones (built volumes, typological characters, landscape systems), in an increasingly automated way, can support the identification and enhancement of historical heritage, classifying and observing its constants in the overall system of urban transformations.

As the interpretation of urban landscape from spatial survey becomes central to describe the dynamics of transformation of the city and its Heritage objects, the integration of multi-source survey data is evaluated to produce an integrated and reliable $3 \mathrm{D}$ database. The comparison of the instrumental datasets in the overall database can be pursued for advancing further 3D modelling experimentations, including opportunities of featurebased semi-automatic recognition. This experimental strategy is intended for enriching the semantic classification from the basis of the survey data, to support the mapping and analysis of the widespread Built Heritage at the urban/territorial scale.

1.2 The case study: heritage location and urban assets within the Cultural Heritage Route of Upper Kama (Russia)

Within the practices of spatial survey, the perception of Heritage sites in settlement contexts can be assigned to the recognition of those qualities characterizing the unique "cultural" valorisation of the architectural object.

In comparison to the different typologies of buildings in an urban context, Heritage objects and monuments are not associated only for their historical correspondence. The cultural label indicates a significance of the site, in the specific context, which finds expression also through geometries, shapes, and 
signs that uniquely qualify the heritage identity. It is the case of characteristic features and traits in planimetric layouts, openings shapes, decorative frames, roof shaping, and other traditional building elements (e.g., onion domes) and layouts (e.g., octagonal o composite volume and planimetric design).

Thus, the feature-based relation within Heritage objects and urban units can be focused on some technical observations:

The variety of geometrical featured and out-of-scale proportions that qualify the monumental assets of Heritage objects (e.g., planimetric polygonal and composite layouts, roof shapes, pinnacles, and domes).

The persistence of Heritage spatial features within centuries, compared to the highly-dynamic changing of urban surfaces and volumes.

The identification of significant paths and routes' connections originally set between different monumental sites, saturated from the planning densification and expansion of contemporary cities.

The last point specifically connects the recognition of heritage qualities to the topic of spatial documentation strategies at the territorial scale, as applicable on Cultural Heritage Routes.
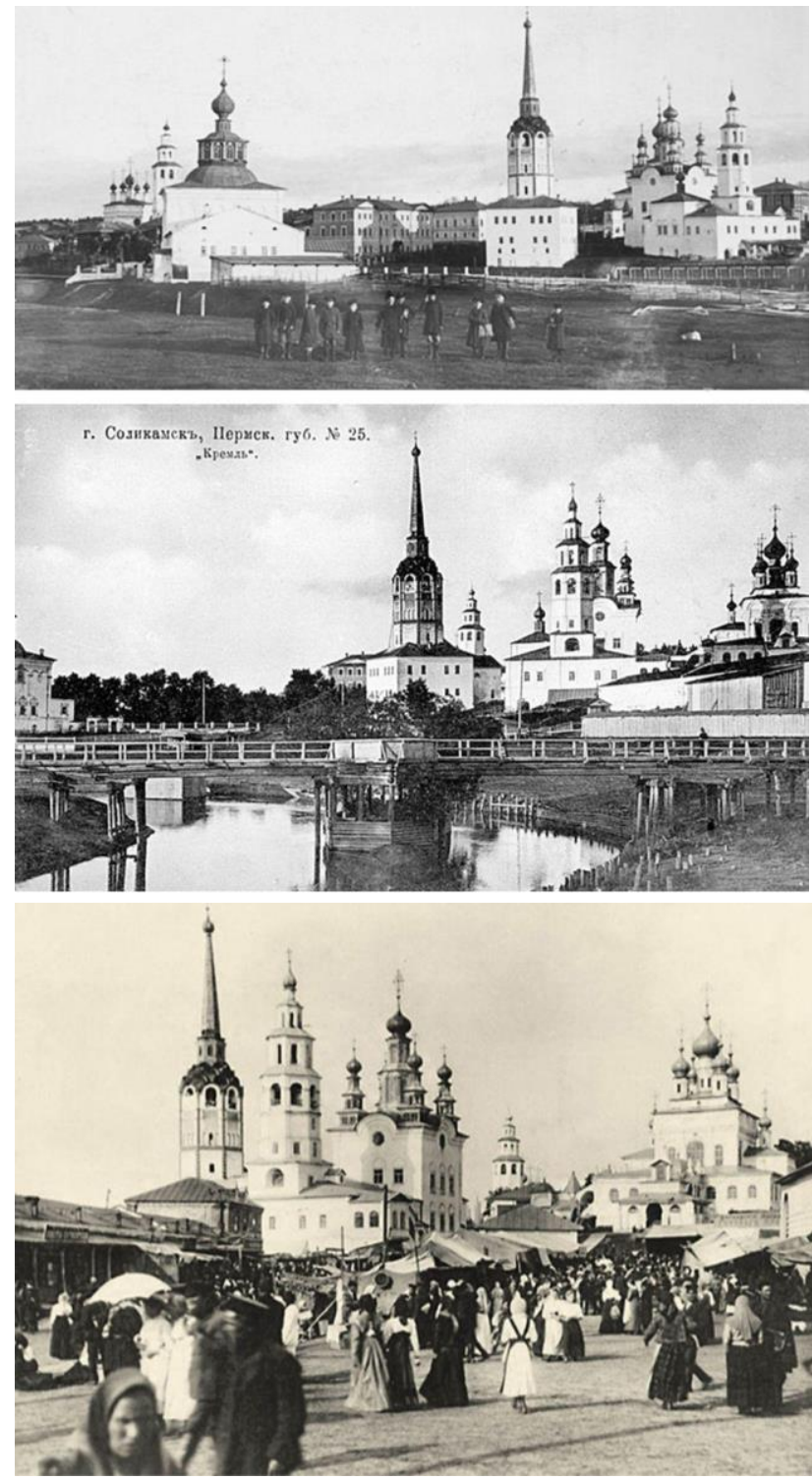

Figure 1. Persistent heritage features within urban saturation phenomena: views of Solikamsk historical center at the end of $19^{\text {th }}$ century, early $20^{\text {th }}$ century, and 1913 .
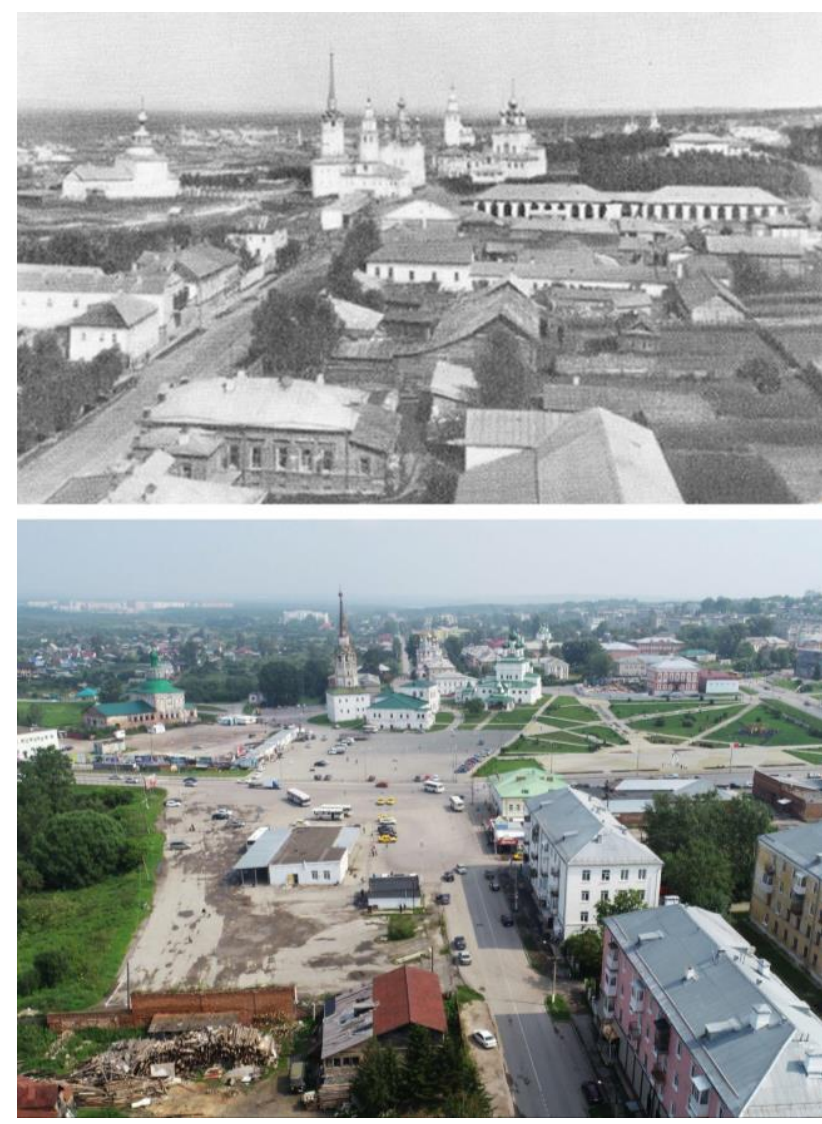

Figure 2. Solikamsk historical center in the $19^{\text {th }}$ century and in 2019: the Heritage objects asset within the urban surroundings.

The pilot case of Upper Kama (Russia) identifies an emblematic Cultural Heritage Route of coexistence between monumental architecture and widespread territorial and urban assets.

The region, developed around the Kama River basin, extends north of Perm Krai for about 7,000 square kilometers, west of the Ural Mountains. Mineral resources and commercial crossroads of the imperial period led Upper Kama to an intense development of industrial settlements between the $15^{\text {th }}-18^{\text {th }}$ centuries. The discovery of local salt mines by merchant families has decreed the development of numerous urban centers, and the flourish of their monumental sites, associated with the districts of Solikamsk (1430), Cherdyn (1535), Usolye (1606). The main heritage buildings located in the historical center of Solikamsk were constructed between 1684 and 1714, in the period of greater economic development.

In the second half of the $18^{\text {th }}$ century, Solikamsk lost its trade importance, substituted by a new Siberian route. The urban asset was modified, replacing the medieval planning structure with a geometric rectangular grid. This layout, preserved till the present days, set the basis for further plans of management and expansion of the urban area, while the placement of the main Heritage buildings was maintained within the changing infrastructures and partitions. (Fig.1)

From the Soviet period, the city has grown into one of the major industrial centers of the Urals, and the development of infrastructures and settlement expansions has declined the relevant role played by Heritage sites in the Upper Kama cultural basin. Many interventions were applied to those urban structures in relation to the Heritage built objects, such as porches, galleries, squares, and paths in the surrounding properties. Some restyling interventions on roofs, domes, and secondary blocks were also applied. (Fig.2) 
The historical development of Solikamsk urban center highlights an uncontrolled management plan of the city within the respect boundaries of its Heritage objects and their traditional characters. The correlation within the reality of the Cultural Heritage Route enhances, in a deeper way, the requirement for monitoring strategies of the evolving changes of territory in relation to their effect on built Heritage recognition. The preservation of the cultural relationship between the historic ensemble and its management in the administration of the territory is directly reflected in the perspectives of attraction and valorization of the Upper Kama route.

The ongoing research activities of the European project H2020RISE-PROMETHEUS are developing a documentation and representation protocol for the Cultural Heritage Routes starting from Upper Kama as a pilot case. The documentation process recovers the Upper Kama classification of heritage scales: "sites", as fragments of historical planning and development of settlements, including monuments and/or ensembles into their boundaries; "ensembles", groups of isolated or joined monuments, buildings and facilities of different destination; "monuments", as separated constructions, building structures and facilities.

Heritage objects scales are evaluated on their datasets from multi-instrumental applications of spatial survey, observing the resolution of point clouds to quantify the geometrical detail of further results. The possibility of recognizing qualifying features from their 3D processing, adopting reality-based mesh models, is pursued to support the identification of Heritage features in the spatial tools of territorial mapping.

\subsection{State-of-art in "intelligent" processing of spatial data about heritage and urban features}

The recent research on the topics of digitization \& information in urban contexts (Schrotter \& Hürzeler, 2020) is increasingly directing the purposes of spatial survey on the identification of sectoral qualities, moving from environmental to historicalcultural ones (Parrinello et al, 2020). Their attribution considers also the analysis of transversal features that, in the cultural or architectural field, can support the interpretation of landscapes and heritage objects, as geometrical and morphometric characters connected to historical phases (Balzani, 2017).

The mapping of settlement areas, at different levels of density and building layout, is largely encouraged in historic centers towards multi-instrumental protocols (El-Hakin et al. 2004), for both aerial and terrestrial acquisition, with appropriate assessments of reliability and resolution in the complementarity between data (Parrinello \& Picchio, 2019). However, the representation of urban scenes from direct processing of spatial data defines a critical point, regarding the extension of input datasets and the resolution of acquired data (Nguatem \& Mater, 2017). In the case of the scale of urban aggregates, the existing strategies contemplate more automated procedures as less formal detail (i.e., vertical surfaces, openings, decorations) is expected in the resulting models.

The simplification of the automated process for a "Manhattan" urban model (Li et al., 2016) cannot be replicated in the case of historical areas, but it can be declined on those relevant features found in the study of the specific Cultural Heritage asset. Its declination on built heritage must include characters of historical and cultural permanence attributed to the monumental elements, in relation to the urban changing/expansions (Balzani \& Maietti, 2018), and on minimal resolutions required for their morphometric identification.

The opportunities for categorizing and segmenting the historical city elements, at different modes and levels of automation, can be pursued also considering feature region-based processes from multi-source point clouds (Nguyen \& Le, 2013). The declination of the specific qualities of urban data (Ennafii et al., 2018) can be applied to ongoing experiments of benchmarks for point cloud segmentation (Matrone et al. 2020). In the same way, the approach to feature-regions recognition on high-poly mesh models (developed in the case of reality-based data for triangulation) can contribute to the scientific topic as an alternative scenario to the machine-learning approach for semiautomatic segmentation (Teruggi et al., 2020).

The intelligent processing of spatial data can be set on appropriate levels of knowledge and perception of heritage and urban features characterizing historic centers, as a pre-required basis for heritage monitoring at the urban scale, and to foresee the development of information systems (GIS, BIM, CIM) on the city and its Cultural Heritage (La Russa et al, 2021).

\section{DIGITAL METHODS OF SPATIAL SURVEY AND ANALYSIS OF DATA RESOLUTION}

Regarding the research case study, the historical center of Solikamsk and its main monumental ensemble, a multiinstrumental survey campaign has been applied in 2019. The main aim has been to maintain a uniform and high metric quality of the architectural data (compatible with the instrumental application), integrating several close-range digital mapping and measurement applications.

The morphological variety of the area, combined with the fast survey requirements, has led to the development of an integrated acquisition plan from the ground level, involving 2 LiDAR instruments, a Terrestrial Laser Scanner TLS (model FARO CamS150), and a Mobile Laser Scanner MLS (model Stencil KAARTA). The integration of photogrammetric data from UAV aerial acquisition (model DJI Phantom Pro4) has been applied to complete the overall data coverage of the site.

\subsection{On-site campaigns: instrumental applications and acquisition strategy}

The main survey has been performed through a TLS LiDAR campaign. A double data acquisition strategy was implemented, considering scan positions both "distributed" along the polygonal paths/parallel to the secondary urban fronts, and "concentrical" to monumental elements of the urban landscape. It has been followed by an acquisition phase through MLS LiDAR, conducted on acquisition trajectories along to urban directories, and through UAV, planning both "grid" (for the urban context) and "point of interest" (for the heritage sites) aerial flight plans. The completeness of acquired data has been processed with a registration phase for each dataset. TLS and MLS point clouds (referenced through morphological targets and cloud-to-cloud alignments) have been integrated with the 3D dense clouds coming from UAV photogrammetric alignment, in terms of both morpho-metric and colorimetric RGB information (from TLS and UAV datasets).

The "concentrical" survey paths were carried out concerning the buildings of historical-cultural interest, identifiable with the main religious architectural blocks. The use of closed polygonal paths for TLS scanning activities (collecting 76 scans, with an average density of $20 \mathrm{mln}$ points/sqm) allowed the reduction of overall metric misalignment, without control targets (e.g., B/W targets) in favor of the fast urban survey. TLS scans included RGB data, extending the acquisition times but allowing more detailed information and shape recognition, especially in the analysis of the decorative elements, as well as in the mapping of the conditions of decay and conservation of the surfaces.

Furthermore, the spatial limit in the acquisition by TLS of large open areas of vegetation assembles from the terrestrial level, as 
well as for elements placed on the top of buildings (i.e., roofs, decorations, and domes), needed the integration of data from an aerial photogrammetric survey application. (Fig.3)

An SfM acquisition strategy concerned 10 flight plans (100-200 photos each one). The first phase included the execution of grid flight plans ( 3 plans, with nadiral and double $45^{\circ}$ camera angles during photos shooting), to allow an overall acquisition of the urban documentation area. The second phase involved 5 pointof-interest flight plans, performed for each monumental building of the historical site with concentric multi-altitude trajectories (with $45^{\circ}$ camera angle), completing a "cone" coverage around the target object according to the height and specific location of each building. The obtained data (High dense point cloud with an average density of $5 \mathrm{mln}$ points/sqm) has permitted to process a unique 3D dataset of the urban area, supporting the reference of the other datasets at the territorial scale. However, the SfM dense cloud, completed of RGB data, presented a reduced morphological resolution on the architectural detail of both Heritage objects and urban assets, due to the high shooting distance (20-50 meters) and, therefore, the photographic sources' resolution $(1$ pixel $=20-60 \mathrm{~cm})$.

Finally, MLS acquisition was developed in trajectories with an average distance of 5-8 meters from the building surfaces. The data presents a lower average density (12 $\mathrm{mln}$ points/sqm), without RGB information, but with competitive shorter times of acquisition (1/10 of TLS survey time) at the urban scale.
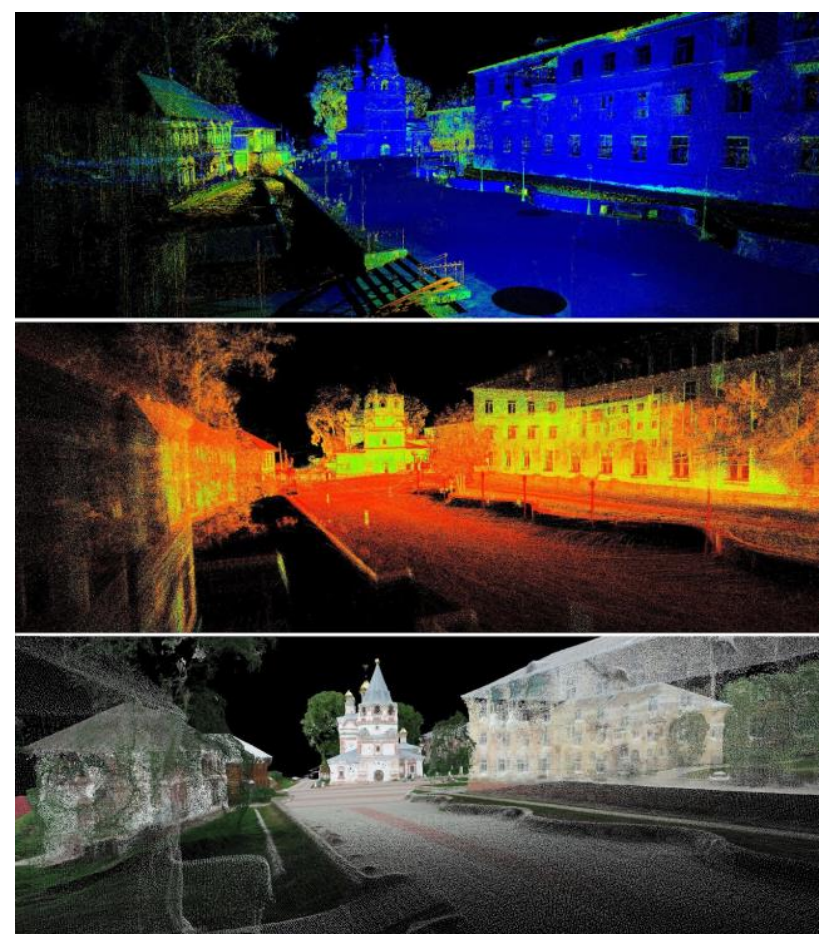

Figure 3. Comparison of point clouds' spatial quality between TLS, MLS, and UAV referenced datasets in Solikamsk center.

\subsection{Reference and reliability of survey data}

The post-production processing of spatial survey has included the reference of the instrumental datasets to a joint UCS. The process has been performed defining common morphological targets between the TLS, MLS, and UAV point clouds. (Fig.4) Urban elements with high geometrical recognizability, as well as clear resolution in the point cloud system, have been chosen (i.e., buildings corners, public space shapes), distributed among different heigh levels. An average number of 20 common targets has been considered for each referenced clouds couple.
TLS spatial data guaranteed accurate referencing and uniform metric reliability $(1-2 \mathrm{~cm})$ in the final point cloud. In MLS survey, the data is qualified at both the architectural and urban scale, with fair metric reliability compared to TLS data (average deviations $3-10 \mathrm{~cm}$ ). UAV point cloud presented metric variance of 8-10 cm compared to TLS discrete surfaces. (Fig.5)

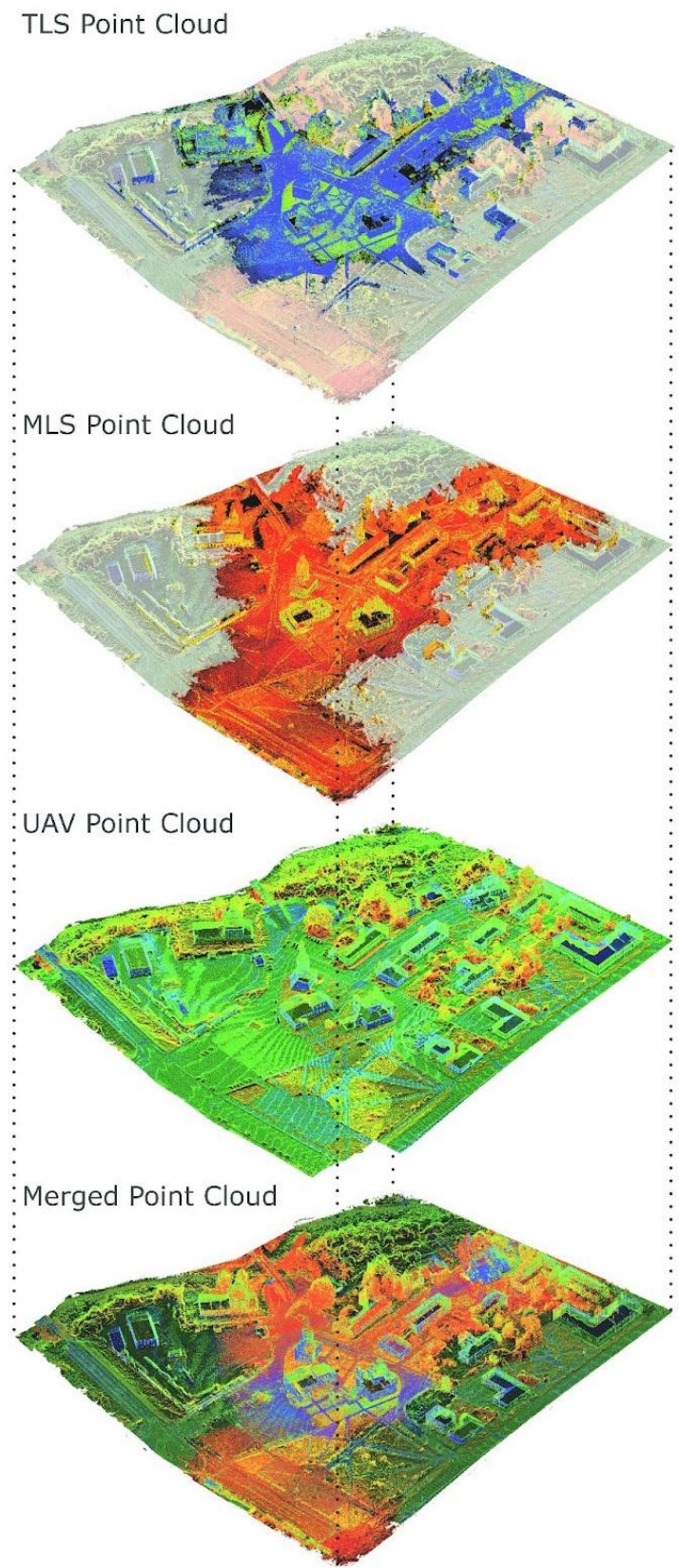

Figure 4. Multi-source urban digital data: complementarity comparison between the referenced instrumental datasets.

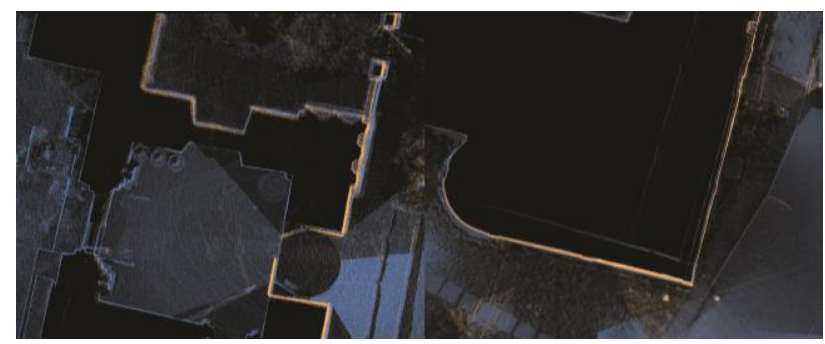

Figure 5. Detail of the correspondence in the alignment between TLS (blue) and MLS (pink) point clouds. 


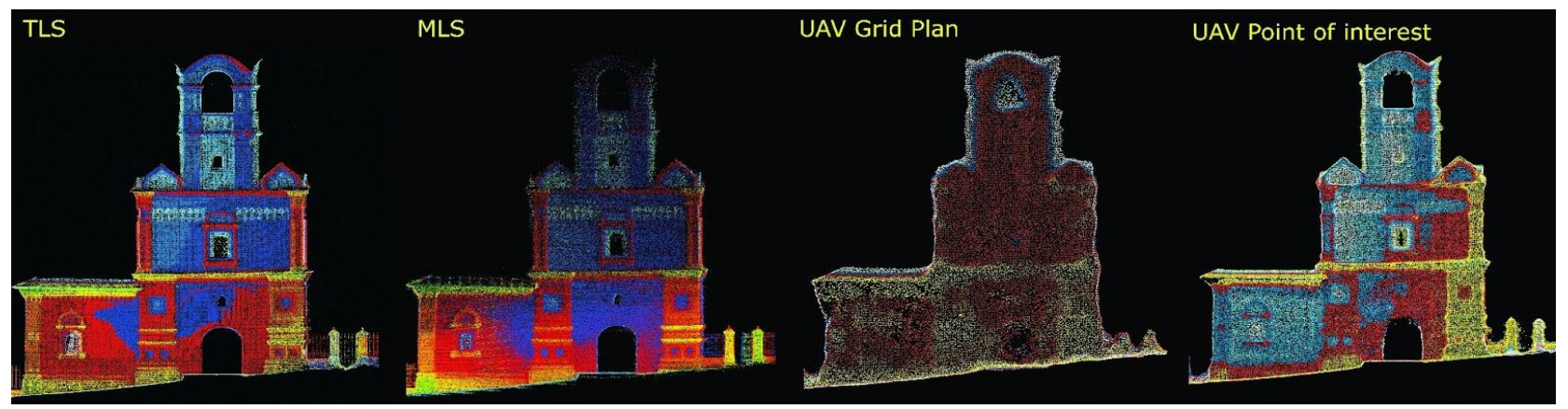

Figure 6. Visualization of morphological quality of spatial data through the "elevation map" view applied to the vertical front of the Church of the Epiphany, one of the Heritage built objects present in Solikamsk historical center.

\subsection{Data analysis and critical report}

A comparative analysis on urban survey spatial data was developed, assuming TLS dataset as the reference basis for instrumental quality.

The visualization of elevation maps, aligned to the vertical reference planes of fronts, has defined a preliminary consideration on the level of geometrical detail acquired by each instrumental dataset, as well as the percentage of survey coverage considering the heights of building levels. (Fig.6) Subsequently, a specific comparison has been developed on localized areas, both for heritage units and urban units surfaces, focusing on the resolution detail in correspondence of opening boundaries (1), decorations (2), and average vertical surfaces (3). The following considerations were achieved (Fig.7):
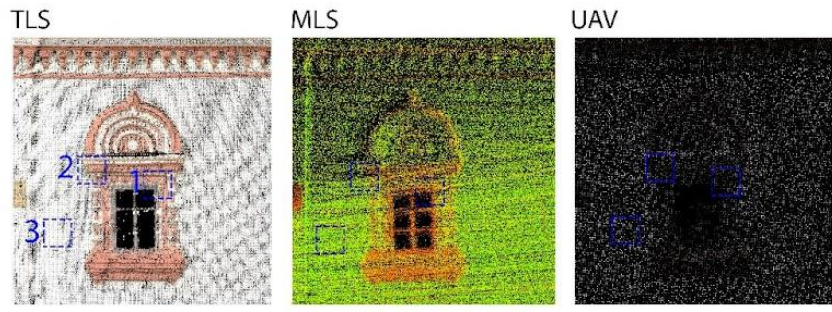

\section{RESOLUTION}
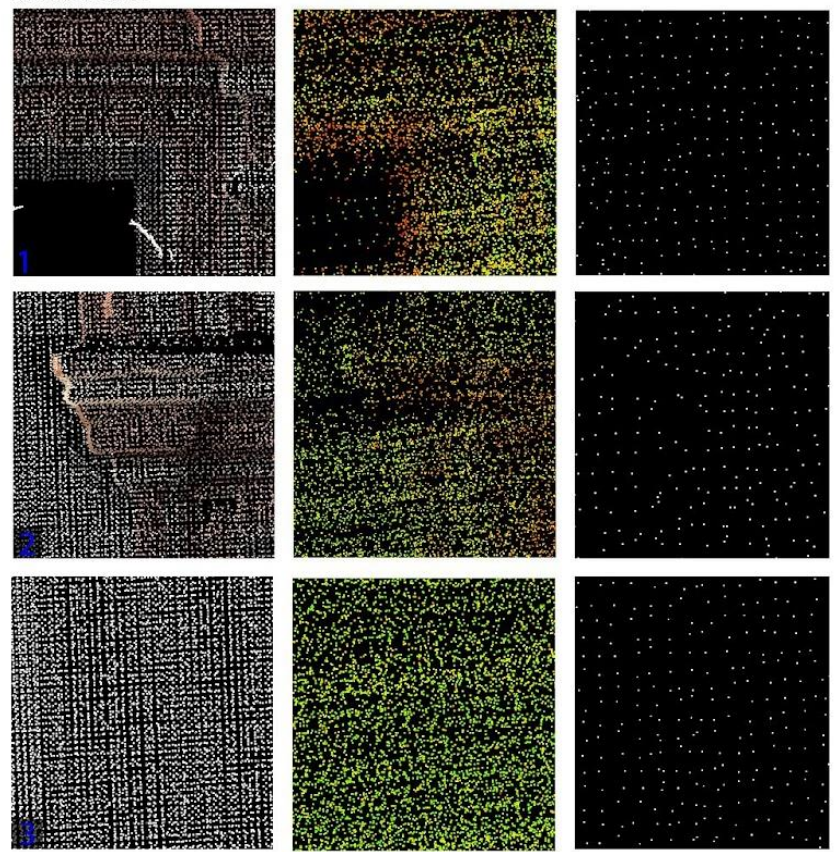

A recognition of main volumetric features has been found in all datasets, also extended to the scale of geometrical features of the architectural systems excepting in the UAV grid plan dataset.

Regarding the resolution on opening borders, LiDARs acquisitions have preserved the recognizance of corners and main shapes, allowing the documentation of openings' distribution both on heritage and urban units. The processing of UAV data on the basis of pixels matching has generated incorrect continuous distributions of points, also in correspondence of openings voids.

Regarding the resolution of decorative apparatuses, UAV grid plan datasets have not achieved a resolution compatible with the minimal geometrical size of morphologies. The same formal system is partially
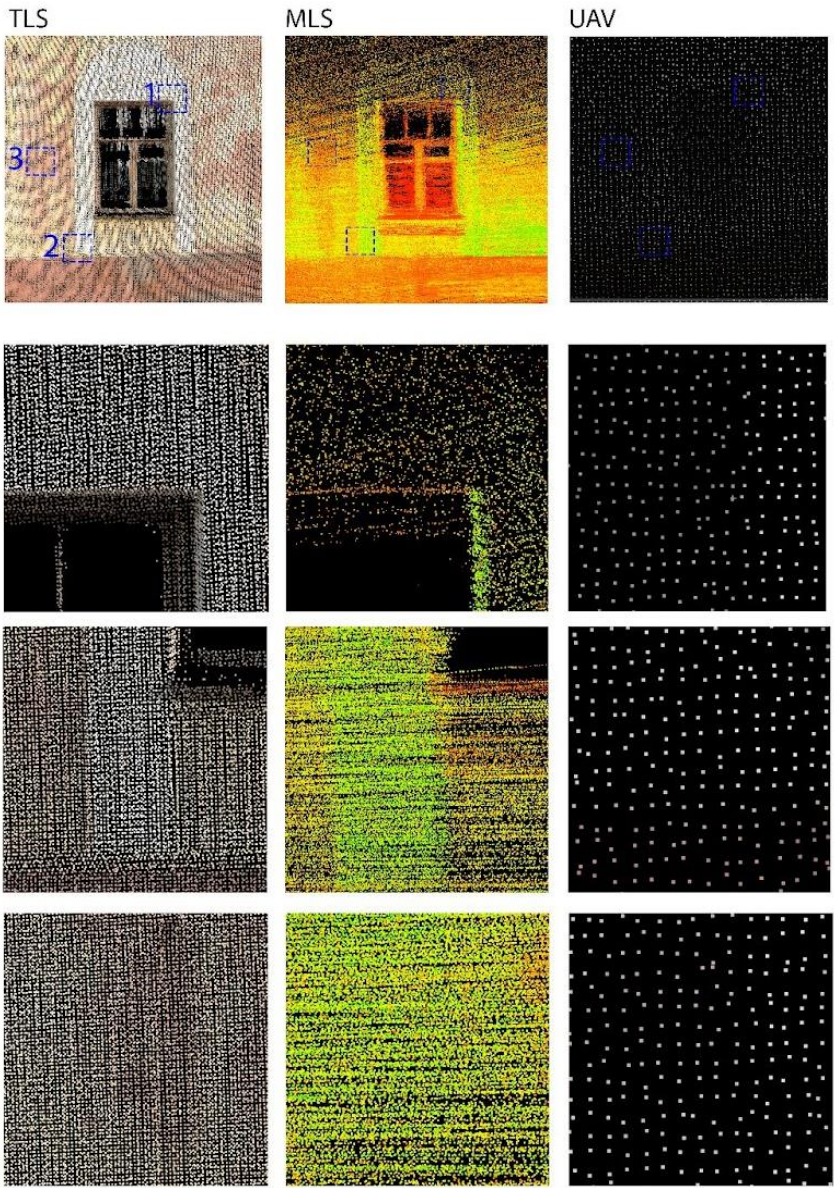

Figure 7. Comparison of resolution quality in spatial data acquired during the urban survey campaign in Solikamsk (2019): two portions from architectural surfaces have been compared, both from an Heritage built object (on the left) and an urban unit (on the right). For each of them, the resolution of detail for the opening bourder (1), the decorative apparatus (2) and the vertical surface (3) has been analysed, considering the different instrumental applications and point clouds' density. 
distinguishable in MLS survey, and well defined in TLS survey dataset.

Regarding the resolution of vertical surfaces, spatial data is correctly interpretable in each dataset. In TLS and MLS datasets, the main axes of LiDAR acquisition are well distinguishable among points grid. This factor is related to the different qualities of resolution in decorative detail, assuming the consideration of the vertical and horizontal distribution of the specific geometries.

In general, the UAV point cloud presented the major critical issues of geometric resolution at the architectural scale (rounded edges, simplified geometric details, architectural voids), while MLS data presented major noise. Resolution properties for each dataset and target spatial quality are summarized in Table 1.

\begin{tabular}{|c|c|l|c|c|l|c|}
\hline \multicolumn{7}{|c|}{ Point cloud resolutions [m] } \\
\hline & \multicolumn{3}{|c|}{ Heritage objects } & \multicolumn{3}{c|}{ Urban buildings } \\
\hline & Open. & Vertical & Dec. & Open & Vertical & Dec. \\
\hline TLS & 0.017 & 0.013 & 0.015 & 0.017 & 0.015 & 0.020 \\
\hline MLS & 0.029 & 0.026 & 0.029 & 0.030 & 0.035 & 0.031 \\
\hline UAV & 0.149 & 0.098 & 0.089 & 0.078 & 0.106 & 0.066 \\
\hline
\end{tabular}

Table 1. Point clouds average resolutions from the compared datasets (TLS, MLS, UAV) on Heritage and urban spatial qualities (openings, vertical surfaces, decorative apparatuses).

\section{3D MESH MODELS AS A BASIS FOR THE RECOGNITION OF BUILT HERITAGE FEATURES}

The overall database of urban documentation on Solikamsk ensemble has been adopted for the experimentation of featurebased extraction processes on high-poly mesh models. The recognition of significant geometrical regions, useful for classification purposes of heritage qualities, has been tested on rough triangulated surfaces from the spatial survey datasets.

\subsection{Meshing pipeline and optimization processing}

A triangulation phase has been processed on spatial survey datasets of Solikamsk urban asset. In order to facilitate the generation of polygonal units of the mesh models, a decimation of the overall point clouds has been performed, according to the average resolutions identified in the critical report of survey data. In this way, a uniform distribution of points/polygonal edges has been ensured in the resulting models.

The same values of average resolution have been assumed as the "Instrumental accuracy" parameter, while the "Geometrical accuracy" parameter of the triangulation has process has been calibrated (in percentage) to find the optimal level for the semiautomated recognition of specific urban/architectural features.

\subsection{Feature-based calibration and automation}

The calibration of parameters in the extraction of feature regions has been optimized considering the following issues: (i) the type of spatial survey dataset (TLS, MLS, UAV); (ii) the average resolution quantified about points distances/polygonal edges; (iii) the expected scale of semantic recognition, depending from the observed details resolutions on the source point clouds and the processed mesh models.

The feature-based recognition process has considered three parameters of calibration. The "Curvature sensitivity" parameter has been adjusted in the range $70-85$, considering the medium geometrical ratio observed within the macro-scale shapes of the mesh models (i.e., rectangular shapes for the volumetric units recognition, polygonal-based solids for the architectural details recognition). The "Minimum recognition area" has been adjusted considering the dimensional scale of feature analysis for each typological quality associated both to heritage and urban units (i.e., fronts' average area for urban qualities, geometric modules units for apparatuses of Heritage objects).

\subsection{Final results}

Final considerations on the features recognition processm based on reality-based mesh models from the spatial survey on the historic center of Solikamsk, have been achieved.

Considering the urban scale, UAV grid plan dataset has proved functionality in the semi-automated segmentation of volumetric units and boundary surfaces. The regions identification has been successful also regarding more complex surfaces belonging to traditional heritage features of monuments (i.e., domes, pinnacles, and polygonal layouts). The same scale of segmentation has been obtained from TLS datasets, while the computation failed on MLS data due to the un-optimized quality of the mesh and the high presence of non-manifold poly-groups (from the highly sparse quality of the source point cloud). (Fig.8)
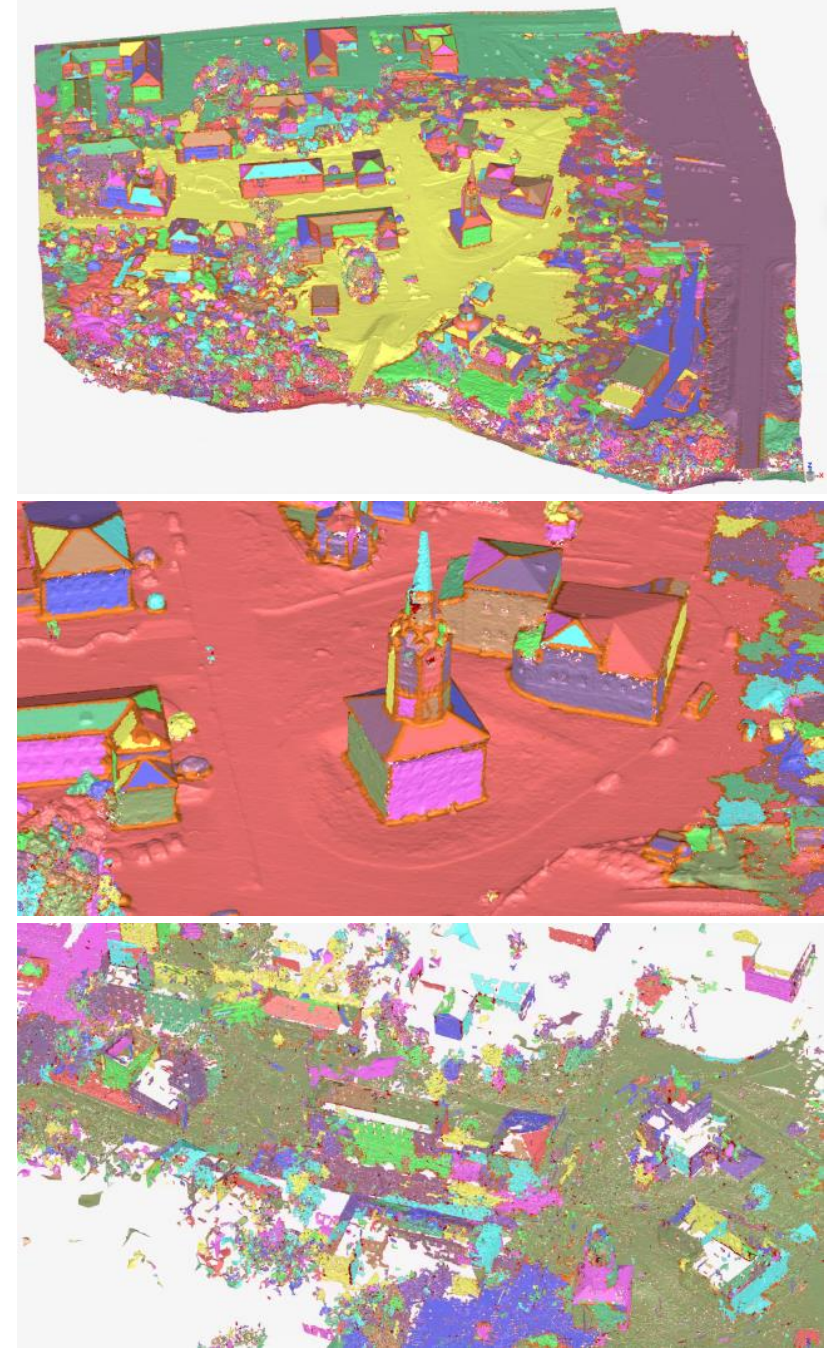

Figure 8. Quality of feature-based semi-automated segmentation on UAV spatial triangulated data (above) and detail on the Heritage qualities of a monumental unit (middle). Similar results from the processing of MLS spatial data (below) 
Considering the architectural scale, UAV grid plan dataset has not permitted a sufficient resolution for the identification of features regarding the geometrical detail qualities of openings and decorative apparatuses. The process has been successful on TLS and MLS datasets, with different performances and qualities of segmentation. In particular, openings features segmentation has been achieved on both TLS and MLS datasets, while the features about decorative apparatuses have been identified only on the TLS dataset. (Fig.9)

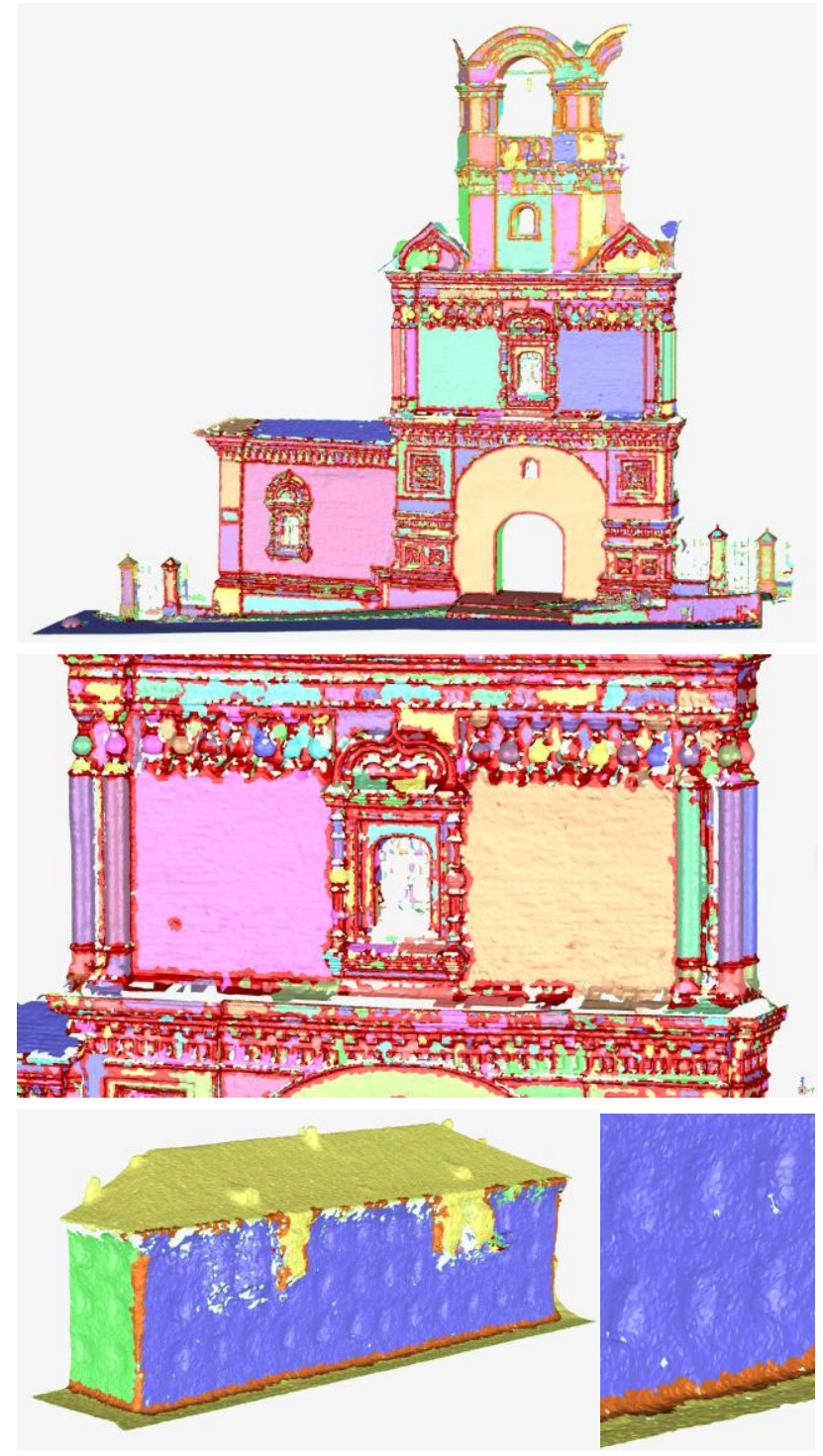

Figure 9. Features recognition for opening and decorative details on TLS spatial dataset applied on Heritage built object main front (above), and opening and decorative apparatus (middle). Failed features recognition of openings on urban fronts in UAV grid plan dataset (below).

\section{CONCLUSIONS}

The objective in the digitization of Solikamsk historical center has regarded the comparison of shapes in spatial datasets and processed 3D models, in order to establish a system of feature regions that qualifies the urban space. Its purpose was for deriving descriptive values and categories useful for the identification and monitoring of Cultural Heritage qualities in built assets also within the dynamics of planning in urban contexts that conceive also Heritage significance.

The comparison of morphometric data has allowed an overall evaluation of the quality of architectural documentation at the urban and territorial scales, improving the planning criteria of the acquisition phases. In particular, more dense points distributions in MLS survey and closer data acquisition to target surfaces in UAV survey are considered for the replicability of the procedure.

The protocol of spatial survey aims to define an associative structure between the urban form and the recognizance of architectural shapes, which can also convey qualities related to Heritage objects. It is not limited to the morphological digitization of the context, but it aims to thematize the analysis of the urban configuration as a projection of cultural values through the conserved features of the historic city. The mapping of formal qualities and characteristics common to Heritage built objects and urban units implies the the development of a catalog of forms and models that condense the cultural and social narrative dimensions of the urban place.

The processes of feature-based recognition on architectural and urban heritage can advance a strategy of evaluation of semiautomated segmentation strategies on 3D point clouds, as opportunities of further analysis on urban survey data, considering the mapping of urban units and the monitoring of heritage spatial relations within territorial planning perspectives.

\section{ACKNOWLEDGEMENTS}

The presented research is developed through the project PROMETHEUS "PROtocols for information Models librariEs Tested on Heritage of Upper Kama Sites" within the European funding program Horizon 2020-R\&I-RISE- Research \&Innovation Staff Exchange Marie Skłodowska-Curie (scientific coordinator Prof. S. Parrinello, University of Pavia). This project has received funding from the European Union's Horizon 2020 research and innovation programme under the Marie Skłodowska-Curie grant agreement No 821870.

The acknowledgment for survey collaboration in Solikamsk historical center is recognized to prof. F. Picchio (University of Pavia) for UAV spatial survey and to dr. A. Dell'Amico (University of Pavia) for MLS spatial survey.

The editorial responsibility of the paragraphs is recognized to $\mathrm{R}$. De Marco for paragraphs 1 and 3, to A. Pettineo for paragraphs 2 and 4.

\section{REFERENCES}

Attene, M., Campen, M., Kobbelt, L., 2013. Polygon mesh repairing: An application perspective. ACM Computing Survevs, 45, 2, 1-6. https://doi.org/10.1145/2431211.2431214

Balzani, M., Maietti, F., 2018. Urban Space and Places of Memory: The Survey as a Tool for Investigating the Process of Transformation. In Graphic Imprints. The Influence of Representation and Ideation Tools in Architecture. Springer, Cham, 541-556.

Balzani, M., 2017. Integrated 3D Morphometric Survey for the Management and Planning of Architectural Heritage. In: Conserving Architecture, AADI Centre, Ahmedabad, 288-305.

Chiabrando, F., Della Colletta, C., Sammartano, G., Spanò, A., Spreafico, A., 2018. "Torino 1911" project: a contribution of a slam-based survey to extensive 3D heritage modelling. Int. 
Arch. Photogramm. Remote Sens. Spatial Inf. Sci, XLII-2, 225234. https://doi.org/10.5194/isprs-archives-XLII-2-225-2018

Colucci, E., Xing, X., Kokla, M., Mostafavi, M. A., Noardo, F., Spanò, A., 2021. Ontology-Based Semantic Conceptualisation of Historical Built Heritage to Generate Parametric Structured Models from Point Clouds. Applied Sciences, 2021, 11(6), 2813. https://doi.org/10.3390/app11062813

El-Hakim, S. F., Beraldin, J. A., Picard, M., 2004. Detailed 3D reconstruction of large-scale heritage sites with integrated techniques. IEEE Computer Graphics and Applications, 2004, 24(3), 21-29. https://doi.org/10.1109/MCG.2004.1318815

Ennafii, O., Le Bris, A., Lafarge, F., Mallet, C., 2021. An efficient representation of 3D buildings: application to the evaluation of City Models. Int. Arch. Photogramm. Remote Sens. Spatial Inf. Sci., XLIII-B2-2021, 329-336, https://doi.org/10.5194/isprs-archives-XLIII-B2-2021-329-2021

Ennafii, O., Le-Bris, A., Lafarge, F., Mallet, C., 2018. Semantic evaluation of $3 D$ city models. HAL, Inria.

La Russa, F. M., Galizia, M., Santagati, C., 2021. Remote sensing and city information modelling for revealing the complexity of historical centers, Int. Arch. Photogramm. Remote Sens. Spatial Inf. Sci., XLVI-M-1-2021, 367-374, https://doi.org/10.5194/isprs-archives-XLVI-M-1-2021-3672021

Li, M., Wonka, P., Nan, L., 2016. Manhattan-World Urban Reconstruction from Point Clouds. In Computer Vision - ECCV 2016. Lecture Notes in Computer Science, vol 9908. Springer, Cham. https://doi.org/10.1007/978-3-319-46493-0_4

Matrone, F., Lingua, A., Pierdicca, R., Malinverni, E. S., Paolanti, M., Grilli, E., Remondino, F., Murtiyoso, A., Landes, T., 2020. A benchmark for large-scale heritage point cloud semantic segmentation, Int. Arch. Photogramm. Remote Sens. Spatial Inf. Sci., XLIII-B2-2020, 1419-1426, https://doi.org /10.5194/isprs-archives-XLIII-B2-2020-1419-2020

Nguyen, A., Le, B., 2013. 3D point cloud segmentation: A survey. In: 2013 6th IEEE Conference on Robotics, Automation and Mechatronics (RAM), 225-230.

Nguatem, W., Mater, H., 2017. Modeling urban scenes from point clouds. In: 2017 IEEE International Conference on Computer Vision (ICCV), Springer, Cham, 54-69, https://doi.org/10.1007/978-3-319-46493-0_4

Parrinello, S., Cioli, F., 2020. Establishment of a Complex Database for the Study of Cultural Heritage through the Reading and Analysis of the Traditional Architecture of Upper Kama. In: Digital Cultural Heritage, Springer, Cham, 51-61, https://doi.org/10.1007/978-3-030-15200-0_4

Parrinello, S., Cioli, F., 2018. Un progetto di recupero per il complesso monumentale di Usolye nella regione della Kama Superiore. Restauro Archeologico, 1/2018, 92-111, https://doi. org/10.13128/RA- 23462.

Parrinello, S., Maksimova, S., Mezenina, K., 2015. Historic Environment Architectural Survey with the use of Digital Technology. Vestnik Perskogo Nacional'nogo Issledovatel'skogo Politehničeskogo Universiteta. Prikladnâ̂
Èkologiâ, Urbanistika, 17, 102-117, https://doi.org/10.15593 /2409-5125/2015.01.07

Parrinello, S., Picchio, F., De Marco, R., Dell'Amico, A., 2019. Documenting the Cultural Heritage Routes. The creation of informative models of historical Russian churches on Upper Kama Region. Int. Arch. Photogramm. Remote Sens. Spatial Inf. Sci., XLII-2/W15, 887-894, https://doi.org/10.5194/isprsarchives-XLII-2-W15-887-2019

Parrinello, S., Picchio, F., 2019. Integration and comparison of close-range SFM methodologies for the analysis and the development of the historical city center of Bethlehem. Int. Arch. Photogramm. Remote Sens. Spatial Inf. Sci., XLII-2/W9, 589-595, https://doi.org/10.5194/isprs-archives-XLII-2-W9589-2019

Parrinello, S., De Marco, R., Galasso, F., 2020. An urban modelling protocol through catalogues and technological modules. From digital survey to 3D information systems for the historic center of Bethlehem. DN, 6, 52-69.

Picchio, F., De Marco, R., Dell'Amico, A., Doria, E., Galasso, F., La Placa, S., Miceli, A., Parrinello, S., 2020. Urban analysis and modelling procedures for the management of historic centres. Bethlehem, Solikamsk, Kotor and Santo Domingo. Urban Design, 2/2020, 102-115.

Schrotter, G., Hürzeler, C., 2020. The Digital Twin of the City of Zurich for Urban Planning. PFG 88, 99-112. https://doi.org/10.1007/s41064-020-00092-2

Teruggi, S., Grilli, E., Russo, M., Fassi, F., Remondino, F., 2020. A Hierarchical Machine Learning Approach for MultiLevel and Multi-Resolution 3D Point Cloud Classification. Remote Sensing. 12(16), 2598. https://doi.org/10.3390 /rs12162598 